\title{
A New Approach to Lumbar Disc Herniation (LDH) Treatment Using Dekompressor Device in Combination with $99.3 \%$ Pure Ethanol
}

\author{
CRISTIAN CONSTANTIN, ANDREEA CINCA², DANA ALBULESCU ${ }^{3}$, CARMEN VALERIA ALBU ${ }^{4}$, MIHAELA MITROI5 $^{5}$, \\ RAMONA CONSTANTINA VASILE ${ }^{6}$, FLORINA NECHITA ${ }^{7}$ \\ IUniversity of Medicine and Pharmacy, Department of Radiology and Medical Imaging, 2 Petru Rares Str., 200349, Craiova, \\ Romania \\ ${ }^{2}$ County Emergency Clinical Hospital, Department of Radiology and Medical Imaging, 1-4 Tabaci Str, Craiova, Romania \\ 3University of Medicine and Pharmacy, Department of Anatomy, 2 Petru Rares Str., 200349, Craiova, Romania \\ ${ }^{4}$ University of Medicine and Pharmacy, Department of Neurology, 2 Petru Rares Str., 200349, Craiova, Romania \\ 5University of Medicine and Pharmacy, Department of Surgery, 2 Petru Rares Str., 200349, Craiova, Romania \\ ${ }^{6}$ University of Medicine and Pharmacy of Craiova, Department of Epidemiology and Primary Health Care Department, 2-4 Petru \\ Rares Str., 200349, Craiova, Romania \\ ZUniversity of Medicine and Pharmacy, Department of Medical Psychology, 2 Petru Rares Str., 200349, Craiova, Romania
}

\begin{abstract}
In the past decade the treatment of lumbar disc herniation has gained more and more popularity throuth minimally invasive procedures. Therefore, we outline a new type of treatment which consists of the combination of two interventional techniques: the utilization of mechanical decompression with the dekompressor device followed by the addition of $99,3 \%$ pure alcohol $\left(\mathrm{C}_{2} \mathrm{H}_{5} \mathrm{OH}\right)$. Furthermore, with a success rate of $100 \%$ in our center, this interventional procedure has proved to be a very effective minimally invasive method in the treatment of lumbar disc herniation.
\end{abstract}

Keywords: Lumbar disc herniation, dekompressor device, chemonucleolysis, pure ethanol, radicular pain

Lumbar pain is one of the most common causes of absenteeism or inability to perform the work tasks. Chronic pain has many effects in the patient's mental system, including anxiety, depression, inability to feel satisfied, negative body image, and an inability to manage daily stress in a healthy manner [1]. Therefore, an important part of the diagnosis and recovery process is the assessment of pain and its effects as well as the risk of addiction and destructive pain managementmethods, such as substance use [2].

Our aim of this study is to outline a new approach for the treatment of lumbar disc herniation used in our center consisted of a combination of two interventional techniques: the utilization of mechanical decompression with the dekompressor device followed by the addition of $99.3 \%$ pure alcohol $\left(\mathrm{C}_{2} \mathrm{H}_{5} \mathrm{OH}\right)$

The final purpose is to evaluate the effectiveness of this technique in the management of discogenic radicular pain through the analysis of two individual cases. These specific cases were monitored over a follow-up period 3 months, 6 months, one year and 2 years after the intervention in which the main outcome evaluations were the subjective measurement of pain relief using the Numerical Rating Scale (NRS) and an objective measurement represented by the MRI images before and after the interventional procedure.

Lumbar disc herniation represents a pathology with severe consequences if left untreated. Primarily, it can cause spinal nerve root compression and local inflammatory response. These two phenomenons in association with the anatomical features of the herniated disc and spinal canal can result in a syndrome which consists of low back pain and sciatica. The global estimation concerning the western population is that approximately $80 \%$ of them would suffer of back pain sometime in their lifetime and more than $50 \%$ of these would suffer of radicular pain [3].
Percutaneous disc decompression procedures have been performed with great success in the past. Many percutaneous techniques such as percutaneous discectomy, laser discectomy, and nucleoplasty are also well received [4].

Our study's aim is to evaluate the results of percutaneous disc decompression procedure for lumbar disc herniation performed in our center with our combined technique and illustrate the effectiveness of this procedure in symptomatic patients with lumbar herniated discs.

Our combined interventional procedure is a percutaneous lumbar disc decompression technique which is a minimally invasive procedure that combines the dekompressor and the addition of pure ethanol as the treatment for symptomatic lumbar disc herniation, opposed to open microdiscectomy, which would be the main treatment modality.

\section{Experimental part}

Matherials and metods

In our center of interventional radiology from Craiova we perform a percutaneous disc decompression procedure for for the treatment of lumbar disc herniation consisted of a combination of two interventional techniques: the utilization of mechanical decompression with the dekompressor device followed by the addition of $99.3 \%$ pure alcohol. The most frequent location of disc herniation is lumbar, especialy at L4-L5.

Firstly, advanced lumbar disc herniation is suspected by the presence of lumbar pain which irradiates in lower limb, lombosciatalgy, hypoesthesia and in the degenerative faze there is a motor deficitwith diminuished or abolishment of osteotendinous reflexes and muscular atrophy.

The diagnosis is confirmed by the MRI imaging which describes in detail the existent lessions, exact location and extension and can establish the eligibility for the intervention. 
The medical notes of the 23 patients admitted in the Department of Interventional Radiology of Craiova for the intervention were reviewed retrospectively. Data collected include: Age, gender, presence and duration of radicular symptoms or signs, previous procedures, the level of disc herniation as seen on magnetic resonance imaging (MRI), and clinical assessment (by the same assessor) preprocedure and at 3, 6, 12 and 24 months following the procedure.

The study consisted of 13 women and 10 men aged between 32 and 64 .

The clinical criteria for inclusion in the study were : radicular symptoms and signs for 6 months or more and failing to respond to conservative management (e.g. physiotherapy, analgesia, epidural injection, and selective nerve root blocks). Pain level was measured pre-procedure and post-procedure through the Numerical Rating Scale (NRS) or Numerical Pain Rating Scale (NPRS) in which patients are asked to circle the number between 0 and 10 , 0 and 20 or 0 and 100 that fits best to their pain intensity.

Radiological inclusion criteria were represented by single-level (L4-L5 or L5-S1 level), small-size, contained lumbar disc herniation (that is clinically relevant) with neural compression as seen on a lumbosacral spine MRI, preservation of disc trilaminar appearance, preservation of at least $50 \%$ of the disc height when compared to adjacent discs.

For the procedure is used a SIEMENS Axiom ARTIS dFA Monoplan Angiograph in a hibrid operating room.

Under fluoroscopic guidance the upper vertebra of the disc herniation is identified and the $C$ arm is positioned cranio-caudal.

Local aneshesia is performed with $5 \mathrm{~mL}$ of $1 \%$ xilin injected peridiscal, intramuscular and subcutaneous.

In the first part of the interventional procedure it is used de dekompressor device under fluoroscopy imaging. This device extracts discal material through precised lateral and postero-anterior movements. The procedure is repeated until there is no discal material extracted onto the probes' tip. Afterwards, through the needle is injected $99.3 \%$ pure ethanol. With a rapid motion the needle is extracted and local compression with a bandage is performed.

Postprocedural, over the next 4 hours the patient is at bed rest and an intramuscular NSAID is administred, being able to be released the same day. It is recommended that any kind of effort that might put pressure on the spine to be avoided.

\section{Results and discussions}

To our knowledge, this is the first published case report regarding this new combined technique used with a success rate of $100 \%$ in our center.

A substance recently made available exploiting the chemical properties of pure ethanol is DiscoGel ${ }^{\circledR}$, which is a radiopaque gelified ethanol more viscous than absolute alcohol. This substance has some important advantages.

\begin{tabular}{|l|l|}
\hline Rating & \multicolumn{1}{|c|}{ Pain Level } \\
\hline 0 & No Pain \\
\hline $1-3$ & $\begin{array}{l}\text { Mild Pain (nagging, annoying, interfering little with } \\
\text { ADLs) }\end{array}$ \\
\hline $4-6$ & Moderate Pain (interferes significantly with ADLs) \\
\hline $7-10$ & Severe Pain (disabling; unable to perform ADLs) \\
\hline
\end{tabular}

This is a radiopaque gelified ethanol which has the same chemical structure as absolute alcohol. In addition, it presents two highly advantageous additives in clinical practice- ethylcellulose, which turns the alcohol solution into a gel being more facile to control and tungsten -a metal which makes the product opaque turning visible. Therefore, the amount of gel injected can be monitored in real time by radioscopy [4].

On the other hand, there are numerous advantages of utilization of $99,3 \%$ pure alcohol which is used with great success in our center of interventional radiology.

It is a well-known fact that ethanol has lytic and necrotizing properties on biological tissues and it has been used for an extensive period of time in interventional procedures. Ethanol produces a molecuar scission of proteoglycans and glycosaminoglycans of the nucleus pulposus which leads to a loss of water-retaining capacity of these components. Therefore, the dehydration and chemical decompression of the disc occurs with the alleviation of the nerve root compression caused by the herniation [5].

Riquelme et al. demonstrates in his study published in 2001 the advantages of using pure ethanol over chymopapain, which was the main nucleolytic agent used in the past. The main benefits include no local septic complications, no aseptic inflammatory complications, no allergic reactions, no major pain after treatment, no chemical discitis or intersomatic disc narrowing and a shorter recovery period for patients [6-7].

Nucleolysis with ethanol is considered to be a very effective, safe and low cost treatment that manages to treat several locations during the same procedure. Furthermore, can be repeated several times. Nucleolysis with pure ethanol can be performed in safe conditions and to certain patients who refuse surgical treatment and have allergic background [4].

In literature, there are only a few studies conducted using pure ethanol due to its' drawbacks : it is not radiopacque and is injected blindly [4]. But in our center we use this method with great success as we wish to demonstrate its effectiveness through this study.

NPRS is a well-known parameter used for the subjective diagnosis of radicular pain in patients with herniated disks. This is a numeric version of the visual analog scale(VAS) where the patients chooses a whole number $(0-10$ integers) which reflects the best the intensity of his/her pain. The most used format is a horizontal bar.

Every patient was asked to make three pain ratings and form an average, corresponding to best, worstand current pain- measured at a certain moment after admission and before the intervention, all measurements aquired over an interval of $24 \mathrm{~h}$ prior to the procedure.

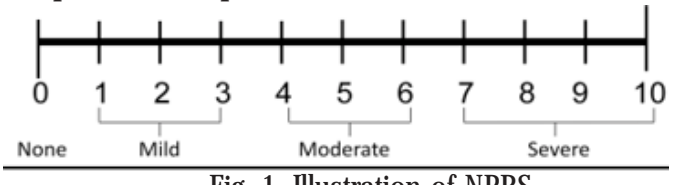

Fig. 1. Illustration of NPRS 


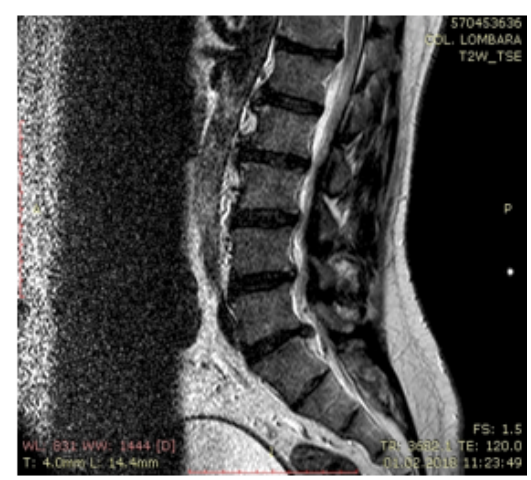

Fig. 2. MRI aspects preprocedural: Sagittal T2W large disc protrusion at the L5-S1 level

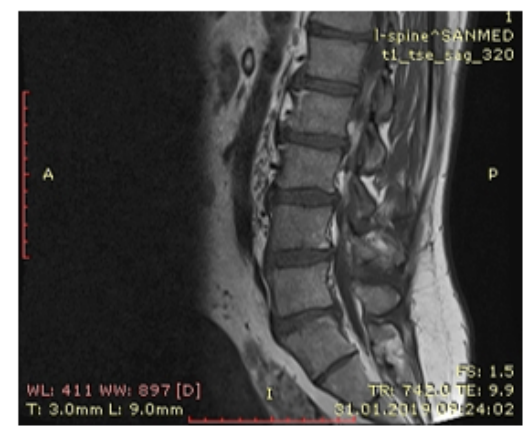

Fig. 3. MRI aspects postprocedural (after 6 months): Sagittal T1W large disc protrusion at the L5-S1 level wich showed significant resolution after decompression and pure alcohol addition (chemonucleolysis with ethanol)

Patient Instructions (adopted from (McCaffery, Beebe et al. 1989): Please indicate the intensity of current, best, and worst pain levels over the past $24 \mathrm{~h}$ on a scale of 0 (no pain) to 10 (worst pain imaginable).

Preprocedural, NPRS shows in all 23 patients a pain mean pain level of 9/10. All patients patients had follow-up checks of pain level after the procedure. Data shows that immediately after the procedure the pain subsided and at $3,6,12$ and 24 months data shows a mean pain level of $0 /$ 10.

Objectively, the success of the interventional procedure was demonstrated through the MRI imaging pre-procedural and post-procedural.The treatment was successful as far as all included criteria are concerned. The Numerical Rating Scale (NRS) measurements show improvement in pain of all patients (100\% success rate). Among all patients with a successful outcome, results were excellent. Moreover, no treatment-linked complications occurred in any patient making this combined technique of mechanical and chemical (represented by ethanol) a successful method in the treatment of lumbar disc herniation.

\section{Conclusions}

Lumbar disc herniation is an important clinical phenomenon, usually based on symptomatology and objective MRI imaging. The overall prognosis of recovery from symptomatic disc herniation is relatively good, and the natural absorption of disc material typically evolves over 1 year, therefore is important to observe the evolution of the procedure over an extensive follow-up period.

Through the presented study we outlined a new approach for the treatment of lumbar disc herniation used in our center consisted of a combination of two interventional techniques: the utilization of mechanical decompression with the dekompressor device followed by the addition of $99.3 \%$ pure alcohol. The results through the two cases presented demonstrate the effectiveness of this technique performed with great success in our center.

There is a rising prevalence of chronic low back pain that is expected to rise with an aging population, therefore new therapies are needed to fill the current gap between conservative measurements and surgical interventions.

Pure ethanol is a safe, low cost substance that can be used with high success rates in the treatment of lumbar disc herniation.

\section{References}

1. ANDERSSON, G.B. The lancet. 1999 Aug 14;354(9178):581-5.

2. SAVAGE, S.R. J ournal of Pain and Symptom Management. 1993 J ul 1;8(5):265-78.

3. OGBONNAYA, S., KALIAPERUMAL, C., QASSIM,A., O'SULLIVAN, M. J Nat Sci Biol Med. 2013 Jan-J un; 4(1): 187-190.

4. THERON, J., GUIMARAENS, L., CASASCO, A., SOLA, T., CUELLAR, H., COURTHEOUX, P. J Spinal Disord Tech. 2007 Oct;20(7):526-32 5.PUENTEDURA, E.J ., BROOKSBY,C.L., WALLMANN, H.W., LANDERS, M.R. J Orthop Sports Phys Ther. 2010 Apr;40(4):214-24.

6. HAEFELI, M., ELFERING, A. Eur Spine J. 2006 J an; 15(Suppl 1): S17S24.

7. CALBOREAN, V., CIOBANU, D., MIREA, S.C., GALCEAVA, O.,GHEORMAN, V., PADUREANU, V., FORTOFOIU, C.M., FORTOFOIU, M., MITA, A., DINESCU, S.N., MISCOCI, S.A., DINESCU, V.C. Benefit of Cardiac Resynchronization Therapy in Patients with Heart Failure. Rev. Chim. (Bucharest), 69, no. 9, 2018, p.2461-2464.

Manuscript received: 30.10 .2018 